\title{
Meta
}

Journal des traducteurs

Translators' Journal

GILE, DANIEL (2005) : La traduction : la comprendre, l'apprendre. Paris: Presses universitaires de France, 278 p.

\section{Chantale Marchand}

Volume 56, numéro 3, septembre 2011

URI : https://id.erudit.org/iderudit/1008345ar

DOI : https://doi.org/10.7202/1008345ar

Aller au sommaire du numéro

Éditeur(s)

Les Presses de l’Université de Montréal

ISSN

0026-0452 (imprimé)

1492-1421 (numérique)

Découvrir la revue

Citer ce compte rendu

Marchand, C. (2011). Compte rendu de [GILE, DANIEL (2005) : La traduction : la comprendre, l'apprendre. Paris : Presses universitaires de France, 278 p.] Meta,

56(3), 731-733. https://doi.org/10.7202/1008345ar

Ce document est protégé par la loi sur le droit d'auteur. L'utilisation des services d'Érudit (y compris la reproduction) est assujettie à sa politique d'utilisation que vous pouvez consulter en ligne.

https://apropos.erudit.org/fr/usagers/politique-dutilisation/
Cet article est diffusé et préservé par Érudit.

Érudit est un consortium interuniversitaire sans but lucratif composé de l’Université de Montréal, l’Université Laval et l’Université du Québec à Montréal. Il a pour mission la promotion et la valorisation de la recherche. https://www.erudit.org/fr/ 
l'impasse dans laquelle il se trouve" (p. 4-5). One can only wonder how does the author conceptualize rationalism, how are "rationalist Muslims" defined, and on what basis she claims that "all" the moderate and rationalist Muslims strive to achieve a re-reading of the Qur'an, especially that the "Muslim world" is not a monolithic entity but is made up of over a billion people coming from very different cultural and socio-political contexts. More importantly, Dib's underlying belief that the situation of the Muslim woman is a direct result of the interpretation of the Qur'an (p. 1-2), reflects the author's adherence to what Lazreg (1994: 13) calls "the religion paradigm" whereby contemporary feminists center their criticism of the situation "of native women [...] on Islam." Lazreg aptly argues that such a paradigm "is steeped in a dual intellectual tradition, orientalist and evolutionary, resulting in an ahistorical conception of social relations and institutions" (Lazreg 1994: 13).

Finally, the choice of the book's title and the cover image is rather infelicitous. The title "D'un islam textuel vers un islam contextuel," does not stand much scrutiny insofar as the very thesis of the book suggests that Islam has always been contextual and never textual. Besides, the cover image of a veiled woman runs contrary to the book's objective, which is to contribute to the emancipation of the Muslim woman by offering a feminist modernist reading of the Qur'an. Indeed, portraying the Muslim woman as veiled contributes to her fetichization and dehistoricization since, unlike the general perception in Western media, "not all Muslim women feel compelled to resort to dress [...] to signal their adherence to Islam and to the Muslim component of their identity" (El-Solh and Mabro 1994: 1). Consequently, the choice of such an image, whether it was dictated by the publisher or made by the author, perpetuates the very monolithic reading of the Qur'an and Islam that the book decries.

These limitations notwithstanding, Dib's work remains a commendable contribution to a subject of critical importance, albeit not within Translation Studies. At a time when the interpretation of the Qur'an is hijacked both by neo-imperial powers to control Muslim-majority countries on the pretext that Islamic teachings are misogynistic, and by Muslim fundamentalists in their striving to control women, it becomes urgent to re-consider the interpretation of the Qur'an and how it reflects on gender as well as on political and social practices.

SanaA Benmessaoud Université de Montréal, Montréal, Canada

\section{REFERENCES}

BAker, Mona (2007): Reframing Conflict in Translation. Social Semiotics. 17(2):151-169.

El-Solh, Camillia F. and Mabro, Judy, eds. (1994): Muslim Women's Choices: Religious Belief and Social Reality. Oxford: Berg Publishers.

Holz-MäntTäRI, Justa (1984): Translatorisches Handeln. Theorie und Methode. Helsinki: Academia Scientiarum Fennica.

Lazreg, Marnia (1994): The Eloquence of Silence. New York: Routledge.

Mason, Ian (1998): Communicative/Functional Approaches. In: Mona BAKER, ed. Routledge Encyclopedia of Translation Studies. London: Routledge, 29-33.

Pym, Anthony (2004): On the social and the cultural in Translation Studies. Visited on 15 February 2010, <www.tinet.org/ apym/on-line/research _methods/sociocultural.pdf $>$.

ReIss, Katharina (1976): Texttyp und Übersetzsungsmethode. Kronberg: Scriptor.

Snell-Hornby, Mary (1988): Translation Studies: An Integrated Approach. Amsterdam: John Benjamins.

Venuti, Lawrence (1998): The Scandals of Translation: Towards an Ethics of Difference. London/ New York: Routledge.

Vermeer, Hans J. (1978): Ein Rahmen für eine allgemeine Translationstheorie. Lebende Sprachen. 23:99-102.

Gile, Daniel (2005): La traduction: la comprendre, l'apprendre. Paris: Presses universitaires de France, $278 \mathrm{p}$.

Après Basic Concepts and Models in Translation and Interpretation Training (1995), Daniel Gile nous offre un ouvrage entièrement consacré à la traduction. On y trouve encore une fois les concepts et modèles chers à l'auteur et le même souci d'exposer en toute transparence ce qui se passe dans la salle de classe. L'ouvrage, qui se veut à la fois un outil de travail pour les pédagogues et un recueil de renseignements pour les étudiants en traduction, est divisé en huit chapitres. Gile se penche tour à tour sur l'enseignement de la traduction, la qualité, la fidélité, un modèle séquentiel de la traduction, l'acquisition de connaissances ad hoc, les langues de travail, des éléments de didactique et, enfin, des éléments de traductologie.

La macroanalyse des textes à traduire est envisagée sous un angle résolument fonctionnaliste avec un accent important mis sur la loyauté professionnelle et la fidélité. Gile présente ainsi les difficultés auxquelles est confronté le traducteur dans cette quête, lesquelles incluent notamment la prise en compte des «informations personnelles» (lapsus, maladresses, mauvais choix terminolo- 
giques ou régionalismes traduisant l'origine de l'énonciateur) et leur inclusion ou omission selon la fonction du texte. Sous l'intitulé «La qualité de la traduction professionnelle: les fondements» (chapitre II), l'auteur fournit une excellente synthèse des principes de l'approche fonctionnaliste et du concept de skopos en illustrant par des exemples concrets comment les diverses «fonctions communicationnelles centrales» (p. 42) peuvent affecter les choix du traducteur. Il se penche également sur les fonctions de base (informer, expliquer et convaincre) tout en montrant que les intentions du lecteur peuvent elles aussi être diverses; dans le domaine politique, par exemple, celles-ci consisteront parfois à vérifier si la totalité de l'information du texte original se retrouve dans la traduction, quelles informations ont été retenues ou la façon dont celles-ci ont été présentées. Cette approche fonctionnaliste, outre l'influence qu'elle exerce sur les décisions du traducteur, comporte l'avantage de «[1]'éloigner sensiblement [...] d'une stratégie de correspondances linguistiques» (p. 46), observe Gile.

Le traducteur, surtout s'il n'est pas rompu à l'exercice de la profession, a besoin de critères pour évaluer son travail. Gile en fait une liste lorsqu'il aborde la qualité en traduction (chapitre III). Par une équation mathématique, il montre que la qualité correspond à une somme de paramètres textuels et de paramètres extratextuels. Les premiers incluent la correction linguistique, la clarté, la justesse terminologique, les correspondances entre les textes d'arrivée et de départ et la présentation graphique; les seconds comptent quant à eux la rapidité de la traduction, son coût, la qualité du contact avec le traducteur et la disponibilité de ce dernier. Pour chacun des paramètres, il existe un seuil minimum d'acceptabilité, rappelle-t-il. Ce qui revient à dire que l'étudiant doit apprendre à répondre à chacun de ces critères jusqu'à un certain degré et combler ses lacunes au fil de sa formation.

Dans un souci d'ordre pédagogique, Gile a recensé les principales fautes et maladresses des étudiants. Faisant de nouveau appel au «modèle séquentiel de la traduction" (chapitre IV) présenté dans son ouvrage de 1995, il suggère des moyens pour prévenir et corriger celles-ci. Pour éviter les fautes de sens, les apprentis sont invités à analyser le texte à traduire en formulant des hypothèses à propos de l'énoncé de départ. Pour chaque unité de traduction, leur jugement est mis à l'épreuve puisqu'ils doivent déterminer si la formulation choisie est plausible ou si des recherches ad hoc supplémentaires et une nouvelle reformulation sont nécessaires. Un recensement intéressant des principales faiblesses méthodologiques à l'origine de fautes et de maladresses chez les étudiants est fourni au chapitre V. Parmi ces faiblesses, on trouvera notamment: 1) le défaut de voir une acception dans son sens spécialisé, ou encore de considérer le caractère polysémique d'un terme ou les nuances entre ses divers synonymes; 2) le remplacement de termes spécialisés indispensables par des synonymes pour éviter une répétition; 3) l'emploi de termes trouvés dans un contexte similaire à celui de l'original sans vérification ultérieure quant à la concordance de sens; 4) l'omission de tenir compte du destinataire ou du lectorat cible (grand public ou spécialistes, par exemple); et 5) le choix d'un terme exclusivement en fonction de son nombre d'occurrences dans Internet. Les enseignants comme les étudiants pourront profiter de cette liste détaillée qui leur permettra de planifier plus efficacement les stratégies de prévention de fautes. En ce qui concerne l'autoévaluation, une place lui est réservée dans la section du chapitre VII intitulée «Diagnostics et traitements individuels des erreurs et des maladresses » (p. 213). Trois types de fautes y sont examinés: les fautes de sens, les fautes et maladresses de langue ainsi que les faiblesses dans la terminologie et la phraséologie spécialisées. Pour chacune d'entre elles, l'auteur nomme les causes les plus fréquentes et montre comment le problème peut être abordé avec les étudiants. La compréhension du texte original fait quant à elle l'objet du chapitre IV. Gile y expose les divers problèmes à l'origine d'une mauvaise compréhension, notamment une maîtrise de la langue de départ ou des connaissances extralinguistiques insuffisantes, une mauvaise qualité du texte de départ ou des fluctuations d'attention chez le traducteur.

Au chapitre VI, l'auteur se penche sur le risque d'interférences linguistiques, lequel s'accroît, explique-t-il, lorsque plusieurs langues actives (de production) sont en présence chez le traducteur. En prenant appui sur le principe de la disponibilité linguistique, il insiste également sur l'importance pour le traducteur d'entretenir et de perfectionner ses langues de travail. Pour illustrer ce principe, Gile recourt à un de ses outils de prédilection; avec son «modèle gravitationnel de la disponibilité linguistique» (p. 184), inspiré du modèle de l'atome de Niels Bohr, il montre que chaque connaissance (linguistique, syntaxique ou autre) occupe une position (orbite) plus ou moins éloignée du noyau (siège des connaissances les plus accessibles au traducteur) selon sa fréquence d'utilisation. Pour rassurer les polyglottes, on retiendra que la disponibilité linguistique est dynamique et que «l'utilisation d'une langue de travail est un antidote efficace contre son oubli» (p.186). Sur un plan pratique, le perfectionnement de la langue active chez les apprentis traducteurs se fera efficacement, de l'avis de Gile, à l'aide d'exercices d'analyses, de résumés de textes et de dissertations. 
Par ailleurs, s'il est un texte utile pour les étudiants en début de formation, c'est bien celui du chapitre VIII intitulé "éléments de traductologie». L'auteur y propose en effet une synthèse réussie de l'histoire traductologique et de l'état actuel de la discipline en dressant un portrait de ses principaux acteurs. Il prend soin de préciser le rôle charnière de Nida dans le changement vers une perspective axée davantage sur la communication (du transfert linguistique à la fonction du destinataire, avec des concepts tels que l'équivalence formelle et l'équivalence dynamique) et celui de Holmes dans l'établissement d'une taxonomie faisant état des divers champs de recherche en traduction et la création de la discipline traductologique ellemême. Gile explique en outre que l'hétérogénéité de ses membres constitue à la fois la force (interdisciplinarité) et la faiblesse (méthodes différentes et vues divergentes quant aux objectifs de recherche) de la communauté traductologique et fait de la traductologie une discipline «fragmentée» (p. 243).

Recherche de la qualité, éthique et motivation du traducteur, capacité de faire preuve de créativité et d'évaluer son travail: voilà des qualités recherchées par les employeurs ${ }^{1}$. Une formation qui se respecte se doit, bien entendu, d'y accorder une certaine importance. Notons que ces concepts occupent une place prépondérante dans la formation préconisée par Gile. Au sujet de «l'évaluation par le côté négatif», il écrira par exemple: «la méthode présente des inconvénients techniques et psychologiques »; les étudiants, craignant l'erreur, auront moins tendance à faire preuve de créativité, « [ce qui] les priverait d'une partie du plaisir que l'on peut éprouver à chercher, trouver et mettre en œuvre des solutions créatives» (p. 218). En résumé, on pourrait dire que Daniel Gile propose une approche qui puise à la fois aux sources des théories de la traduction, aux tendances en pédagogie et à sa propre pratique de traducteur et d'enseignant. On ne peut qu'apprécier l'authenticité et la générosité dont il fait preuve dans cet ouvrage, et dans ses autres publications sur le sujet. En guise de conclusion, citons un passage de l'auteur extrêmement éloquent à cet égard:

$\mathrm{Si}$, à travers une attitude pédagogique, l'on arrive à faire découvrir aux étudiants le plaisir qui accompagne un travail d'analyse, de recherche et de rédaction, si on peut en limiter les aspects pénibles, on peut espérer leur faire aimer le travail de traduction et contribuer par là à un apprentissage optimal et au maintien de la qualité au-delà de la formation (p. 233).

À méditer...

Chantale Marchand Université de Montréal, Montréal, Canada

\section{NOTE}

1. Álvaro Echeverri (2008) examine en profondeur cet aspect métacognitif de l'apprentissage.

\section{RÉFÉRENCES}

Echeverri, Álvaro (2008): Métacognition, apprentissage actif et traduction: L'apprenant de traduction, agent de sa propre formation. Thèse de doctorat. Montréal: Université de Montréal.

Gile, Daniel (1995): Basic Concepts and Models in Translation and Interpretation Training. Amsterdam/Philadelphia: John Benjamins.

Atelier de traduction Nos 9 et 10 (2008): Dossier. La traduction du langage religieux I et II. Actes du Colloque international «La traduction du langage religieux en tant que dialogue interculturel et interconfessionnel ", 11-13 juillet 2008, Suceava. Suceava: Editura Universitatii, $228+338$ p.

La revue Atelier de traduction est une revue semestrielle réalisée par le Centre de Recherches INTER LITTERAS de la Faculté des lettres et des sciences de la communication de l'Université Stefan cel Mare de Suceava et par les participants aux ateliers de traduction organisés sous l'égide du Service de Coopération et d'Action Culturelle auprès de l'Ambassade de France en Roumanie; Bureau du Livre; Centre culturel français de Iasi Bureau Europe centrale et orientale - AUF - Bucarest.

Le noyau de chaque numéro est un dossier consacré à un débat, par exemple: "Traduction et francophonie», "L'autotraduction», etc., accompagné d'autres rubriques intéressantes, telles que «Entretien», «Pratico-théories», "Vingt fois sur le métier», "Terminologies», "Comptes rendus», "Portraits de traducteurs».

Le dossier des numéros 9 et 10 de 2008 est consacré à la traduction du langage religieux. Ce dossier s'inscrit dans un horizon œcuménique, qui engendre et multiplie les questions plutôt que de donner des réponses nettes relatives à ce carrefour entre religion, philosophie, science et littérature que représente la traduction du langage religieux.

Les discours théoriques laissent souvent la place aux exemples concrets, aux analyses textuelles, présentées la plupart du temps par des traducteurs chevronnés. On apprend donc, tour à tour, à quel point il a été difficile de traduire les auteurs roumains en français ou les auteurs français en roumain. L'implicature est vue comme un trait du texte étranger qui révèle une différence entre les deux cultures, habituellement un manque dans les connaissances du lecteur et que le 